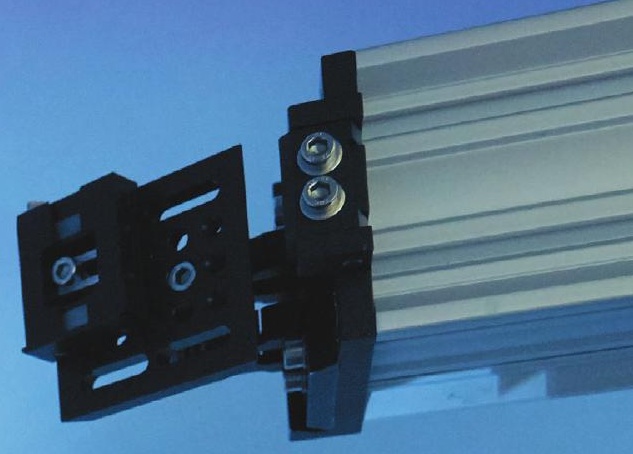

\title{
THE MOUSE INTHE VIDEOGAME
}

A mouse scurries down a hallway, past walls lined with shifting monochrome stripes and checks. But the hallway isn't real. It's part of a simulation that the mouse is driving as it runs on a foam wheel, mounted inside a domed projection screen.

While the mouse explores its virtual world, neuroscientist Aman Saleem watches its brain cells at work. Light striking the mouse's retinas triggers electrical pulses that travel to neurons in its primary visual cortex, where Saleem has implanted electrodes. Textbooks say that these neurons each respond to a specific stimulus, such as a horizontal or vertical line, so that identical patterns of inputs should induce an identical response. But that's not what happens. When the mouse encounters a repeat of an earlier scene, its neurons fire in a different pattern.

"Five years ago, if you'd told me that, I'd have been like, 'No, that's not true. That's not possible," says Saleem, in whose laboratory at University College London we are standing. His results, published last September ${ }^{1}$,

\section{What virtual-reality animal experiments are revealing about the brain.}

\section{BY LIAM DREW}

show that cells in the hippocampus that track where the mouse has run along the hallway are somehow changing how cells in the visual cortex fire. In other words, the mouse's neural representation of two identical scenes differs, depending on where it perceives itself to be.

It's no surprise that an animal's experiences change how it sees the world: all brains learn from experience and combine multiple streams of information to construct perceptions of reality. But researchers once thought that at least some areas in the brain - those that are the first to process inputs from the sense organs - create relatively faithful representations of the outside world. According to this model, these representations then travel to 'association' areas, where they combine with memories and expectations to produce perceptions.

That's now known to be simplistic — a view largely based on twentieth-century studies in which neuroscientists anaesthetized or immobilized animals to measure how their brains reacted to stimuli passively presented to them. In a landmark 2010 study in mice, researchers pushed the study of vision forward: they held the animals' heads still to maintain control of what the mice looked at, but allowed their subjects to stand or run on a ball. In moving mice, visual neurons fired more than twice as fast $^{2}$. That finding triggered a string of experiments showing that how an animal is behaving changes activity throughout its brain, including the way in which sensory areas respond to the outside world. "These results have been transformative," says Anne Churchland, a neuroscientist at Cold Spring Harbor Laboratory in New York. "I think we have only just scratched the surface." 
A mouse runs on a wheel in a virtualreality experiment.

Virtual reality (VR) technology isn't the only way to explore this. Neuroscientists have, for instance, recorded from multiple brain areas simultaneously as an animal moves around in the laboratory. But over the past decade, dozens of groups have begun placing mice and rats inside VR simulations. Saleem says he's often asked if this is necessary research - and his answer is that most of his experiments would otherwise be impossible. In VR, animals have a greater sense of agency, because the worlds they see are programmed to respond to their movements - as in the real world. But the scientist still retains tight control of the experiment, and can add, subtract or warp sensory inputs at will. Parallels with the Matrix films are not lost on Saleem's group: the experimenters named parts of their VR equipment Neo, Trinity and Morpheus, after characters from the films, in which people live in virtual realities.

As long as animals have their heads restrained and experience VR environments that are much simpler than real ones, there will always be questions about the extent to which brains process VR as they do 'real' reality, as researchers put it. But exploring these questions has helped VR researchers to unlock new ways of interrogating how the brain constructs its perceptions of the world. "VR has been a huge stimulus to neuroscience," says Michael Stryker, a neuroscientist who studies vision at the University of California, San Francisco, and who published the pioneering 2010 study.

\section{VIRTUALLY REAL}

As far back as the 1960s, biologists studying movement tethered fruit flies' heads while the insects walked on ping-pong balls. Neuroscientists revived these approaches when VR took off in the early 2000s. The technology offered a way to observe brain activity in animals that could be fooled into thinking they were roaming freely, even though their heads were held still. That allowed the use of intricate brain-recording techniques — such as electrical recordings from inside neurons, or optical microscopy to image large numbers of neurons. Flies were again mounted on balls, or had their heads tethered as they 'flew' in wind tunnels. Researchers studying zebrafish larvae secured the fishes' heads in agar blocks but allowed them to flick their tails as if they were swimming. The zebrafish and fly studies helped scientists to see simpler neural circuits in action. But other researchers wanted to study mammalian brains, like our own, which are more complex; in particular, they were interested in the cerebral cortex, the large, multi-layered sheet of neurons central to mammalian intelligence.

Researchers reported a prototype VR system for rats as early as $2005^{3}$. But the approach went mainstream after a group led by David Tank, at Princeton University in New Jersey, built VR simulators for mice. Tank was studying the mouse's intrinsic navigation system: cells in the hippocampus and nearby brain regions that help an animal to track its position in space. Researchers had first described this system by implanting electrodes in rats moving freely in large enclosures, and recording brain cells firing - advances that secured the 2014 Nobel Prize in Physiology or Medicine. But Tank wanted to work out finer details of how neurons fired by using delicate intracellular electrodes. These required an animal's head to be held still, leaving little for its navigational system to track. So Tank, together with Chris Harvey, now at Harvard Medical School in Boston,

\section{"VRHASBEENA HUGESTIMULLSTO NEUROSCIENCE."}

Massachusetts, developed a virtual world rich enough to create a sense of moving around space. In 2009, the group ${ }^{4}$ reported its mouse VR system in a study that, for the first time, described the inner workings of hippocampal neurons as they created a map of space from multiple streams of sensory inputs.

That research has led to a surge in papers on mammalian VR in the past few years. Labs such as Stryker's adapted Tank's set-up, and - as in Saleem's case - recorded from navigational cells and visual cells to see how they influenced each other. At the Howard Hughes Medical Institute's Janelia Research Campus in Ashburn, Virginia, mice walk in complete darkness on a treadmill; panels flanking the whiskers move in and out to give the animals the impression of moving down a constricting and expanding tunnel ${ }^{5}$. At Northwestern University in Evanston, Illinois, mice follow odours that get weaker or stronger depending on how they run on a spherical treadmill ${ }^{6}$. “There's many versions and varieties," Tank says. "It's a whole ecosystem."

It's difficult to tell how animals experience these virtual worlds. Researchers often use the analogy of a person immersed in a video driving game: they know they are not actually steering a racing car, but their brain is sufficiently engaged in the task to activate the same visual processes that would accompany real driving. Scientists' main concern when interpreting VR experiments is that, although projected images change in sync with a mouse's movement, other sensory inputs, such as changing odours, sounds and what the animal feels through its whiskers, do not. And a mouse with its head fixed receives none of the head movement or balance signals that it would in the real world. These problems are particularly troubling for scientists who want to study how the hippocampal navigation system creates its sense of place using a variety of inputs.
In 2015, Mayank Mehta, a neuroscientist at the University of California, Los Angeles, reported that hippocampal neurons fired differently — and to a lesser extent - when rats explored a 2D VR system, compared with when the rodents walked around a real-world replica room $^{7}$. (Mehta printed patterns on the curtains of the room to recreate his VR set-up.) In the real world, says Mehta, the synchronized changing of tactile, smell and sound cues, together with the rat's ability to move its head and body naturally, engages the animal's navigational system in a different way from in the simulation. But Mehta's points haven't significantly dented interest in VR. As he and others have shown, VR and real-world set-ups generate similar neural firing patterns when rodents move in a straight line down a corridor. And in newer 2D VR systems that allow rodents' bodies to rotate on a spherical treadmill, creating naturalistic balance and movement signals, researchers do see similar neural representations of space in virtual and real worlds.

These experiments are helping researchers to ask at what point virtual reality is so real that the brain can't tell the difference, Mehta says. A related approach is augmented reality, in which animals move freely around a lab space, but in such a way that what they see is controlled - and manipulated - by images projected onto walls ${ }^{8}$. In these studies, scientists cannot use some neural recording methods, but they can see what inputs matter for the brain's perception of space. As long as scientists are aware of these nuances, VR is a powerful technique, says Edvard Moser, a neuroscientist at the Norwegian University of Science and Technology in Trondheim, who shared the 2014 Nobel prize for his work on the brain's navigational system.

\section{IMPOSSIBLE WORLDS}

What makes VR especially powerful, Moser says, is that "you can manipulate factors in the environment as you like, and keep the others constant". Simulations can even seem to change the laws of physics. In nature, if an animal runs at a particular speed, its visual inputs change at that speed. But, in VR, that linkage can be broken.

Neuroscientists have long wanted to know, for instance, whether an animal's sense of moving through space is swayed more by internal signals about its own movement, or by what it sees. Last year, Lisa Giocomo at Stanford University in California looked at neurons that feed into the hippocampus, and showed in a VR experiment that when the outside world is whipping by at great speed - such as when an animal is running through a forest, looking at nearby trees - visual cues dominate this part of the navigational system. When these signals move more slowly, as when an animal is gauging its position relative to a distant mountain range, signals generated by the animal's own movement take over ${ }^{8}$.

Georg Keller, a neuroscientist at the Friedrich 


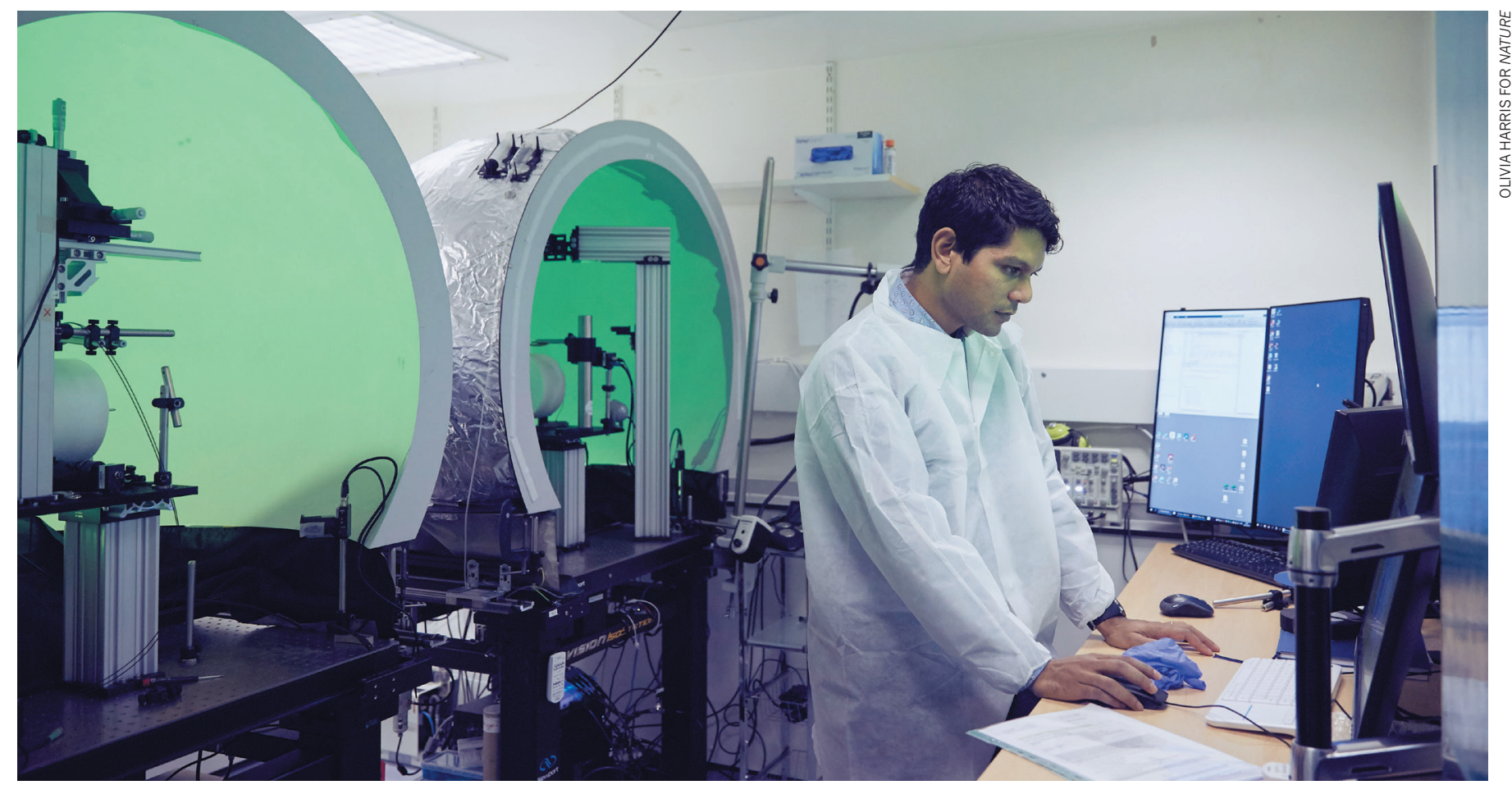

Miescher Institute for Biomedical Research in Basel, Switzerland, also manipulates the connection between a mouse's movement and what it sees. In 2016, he reported that when a small part of a virtual corridor does not move in synchrony with the mouse's movement, a subset of what he calls "mismatch cells" start to fire ${ }^{9}$. Keller thinks these cells help the mouse to detect signals that are not accounted for by the consequence of its own movement. He interprets this as feeding into a long-standing theory, with roots stretching back to the 1940s, that the brain is constantly making predictions about what it expects to encounter, and that cortical areas function to detect deviations from these predictions. In this model, what the mouse anticipates seeing is derived in part from memories of what it has seen, its navigational tracking and its own movement. And this anticipation affects how the neurons in its visual cortex fire.

Other VR experiments show how visual cortical activity is shaped by what an animal has learnt. In 2018, neuroscientist Nathalie Rochefort at the University of Edinburgh, UK, reported that after she began giving mice a reward - a squirt of water to quench their thirst - at a specific point in a virtual corridor, the representation of that corridor in the visual cortex changed dramatically. At first, all regions of the corridor generated equal amounts of neural activity in the visual cortex. But once the 'reward zone' had gained significance, most of the visual cortical neurons started to fire only there ${ }^{10}$.

After decades of detailed work on rodent brains, scientists are also using VR to ask if their findings apply equally to primates that have brains more like our own. Elizabeth Buffalo at the University of Washington,
Seattle, for example, is using VR to show that the primate hippocampus, just like the rodent one, contains 'place cells' that fire in specific locations in a virtual world. In her experiments, monkeys explore projected Y-shaped mazes by moving joysticks. Buffalo tests the monkeys' ability to remember routes around the simulations, so that she can analyse how the hippocampal representation of space intersects with its crucial role in memory ${ }^{11}$. The thing we like about VR is the richness of the behavioural tasks that we can employ," she says, "They open up new questions."

\section{TOWARDS A REALER REALITY}

After a decade of VR research, scientists studying sensory systems are moving away from viewing individual brain areas as being dedicated to processing unique aspects of the world, says neuroscientist David Schneider at New York University in New York City. For a long time, scientists asked how sensory neurons fired in response to sights, sounds, smells and textures. But now, he says, researchers appreciate that neurons in sensory cortices are "representing some intersection of a feature in the world - and some aspect of what an animal is doing". When scientists presented images only to immobilized mice, they understood cells in the primary visual cortex simply to be encoding these visual stimuli. When moving mice viewed moving images, it became clear that movement also shaped the activity of these neurons. Now that scientists are scrutinizing the animals' sense of place, expectations and learnt associations, it turns out that these, too, account for aspects of neural activity in the visual cortex.

Jasper Poort, a neuroscientist at the University of Cambridge, UK, is helping to develop head-mounted camera systems that track a
Neuroscientist Aman Saleem at University College London, with the VR domes he uses in simulations.

are moving around freely, rather than with their heads fixed in place. He predicts that observing animals undertaking more complex behaviours in more complex environments will show that memory, attention and other aspects of brain function affect early sensory processing to an even greater extent. "The way you approach an experiment can give you completely different results," he says.

In 50 years' time, Schneider adds, scientists could look at models built on data from VR experiments, and see them, too, as overly simple. "In the long run, as technology advances, things will get less virtual," says Stryker. But, for now, the mouse in the video game is still a fruitful experiment, he says. "Real reality is hard to work with."

Liam Drew is a science writer in London, UK.

1. Saleem, A. B. et al. Nature 562, 124-127 (2018).

2. Niell, C. M. \& Stryker, M. P. Neuron $65,472-479$ (2010).

3. Hölscher, C. et al. J. Exp. Biol. 208, 561-569 (2005)

4. Harvey, C. D. et al. Nature 461, 941-946 (2009).

5. Sofroniew, N. J., et al. J. Neurosci. 34, 9537-9350 (2014).

6. Radvansky, B. A \& Dombeck, D. A. Nature Commun. 9, 839 (2018).

7. Aghajan, Z. M. et al. Nature Neuro. 18, 121-128 (2015).

8. Campbell, M. G. et al. Nature Neuro. 21, 1096-1106 (2018).

9. Zmarz, P \& Keller, G. B. Neuron 92, 766-772 (2016).

10.Pakan, J. M. P. et al. Cell Rep. 24, 2521-2528 (2018).

11.Rueckemann, J. W. \& Buffalo, E. A. Curr. Op. Behav. Sci. 17, 155-160 (2017). 